\title{
ON THE COMPUTATION OF THE RATLIFF-RUSH CLOSURE, ASSOCIATED GRADED RING AND INVARIANCE OF A LENGTH
}

\author{
AMIR MAFI \\ Dedicated to Professor Tony J. Puthenpurakal
}

\begin{abstract}
Let $(R, \mathfrak{m})$ be a Cohen-Macaulay local ring of positive dimension $d$ and infinite residue field. Let $I$ be an $\mathfrak{m}$-primary ideal of $R$ and $J$ be a minimal reduction of $I$. In this paper we show that if $\widetilde{I^{k}}=I^{k}$ and $J \cap I^{n}=J I^{n-1}$ for all $n \geq k+2$, then $\widetilde{I^{n}}=I^{n}$ for all $n \geq k$. As a consequence, we can deduce that if $r_{J}(I)=2$, then $\widetilde{I}=I$ if and only if $\widetilde{I^{n}}=I^{n}$ for all $n \geq 1$. Moreover, we recover some main results of 4 and 11]. Finally, we give a counter example for Question 3 of 21 .
\end{abstract}

\section{INTRODUCTION}

Throughout this paper, we assume that $(R, \mathfrak{m})$ is a Cohen-Macaulay local ring of positive dimension $d$, infinite residue field and $I$ an $\mathfrak{m}$-primary ideal of $R$. An ideal $J \subseteq I$ is called a reduction of $I$ if $I^{n+1}=J I^{n}$ for some $n \in \mathbb{N}$. A reduction $J$ is called a minimal reduction of $I$ if it does not properly contain a reduction of $I$. The least such $n$ is called the reduction number of $I$ with respect to $J$, and denoted by $r_{J}(I)$. These notions were introduced by Northcott and Rees [20, where they proved that minimal reductions of $I$ always exist if the residue field of $R$ is infinite. Recall that $x \in I$ is a superficial element of $I$ if there exists $k \in \mathbb{N}_{0}$ such that $I^{n+1}: x=I^{n}$ for all $n \geq k$. A set of elements $x_{1}, \ldots, x_{d}$ is a superficial sequence of $I$ if $x_{i}$ is a superficial element of $I /\left(x_{1}, \ldots, x_{i-1}\right)$ for all $i=1, \ldots, d$. A superficial sequence $x_{1}, \ldots, x_{d}$ of $I$ is called tame if $x_{i}$ is a superficial element of $I$, for all $i=1, \ldots, d$. Elias [8] defined and proved the tame superficial sequence exists (see also [6]). Swanson 27] proved that if $x_{1}, \ldots, x_{d}$ is a superficial sequence of $I$, then $J=\left(x_{1}, \ldots, x_{d}\right)$ is a minimal reduction of $I$. It is known that every minimal reduction can be generated by superficial sequence (see [26] or [6]).

2000 Mathematics Subject Classification. 13A30, 13D40, 13 H10.

Key words and phrases. Ratliff-Rush filtration, Minimal reduction, Associated graded ring. 
The Ratliff-Rush closure of $I$ is defined as the ideal

$$
\widetilde{I}=\cup_{n \geq 1}\left(I^{n+1}: I^{n}\right)
$$

It is a refinement of the integral closure of $I$ and $\widetilde{I}=I$ if $I$ is integrally closed (see [23]). The Ratliff-Rush filtration $\widetilde{I^{n}}, n \in \mathbb{N}_{0}$, carries important information on the associated graded ring $G(I)=\bigoplus_{n \geq 0} I^{n} / I^{n+1}$. For example, Heinzer, Lantz and Shah [13] showed that the $\operatorname{depth} G(I) \geq 1$ if and only if $\widetilde{I^{n}}=I^{n}$ for all $n \in \mathbb{N}_{0}$. The aim of this paper is to compute the Ratliff-Rush closure in some senses and as an application, we shall reprove some main results of [4, 12] and [1]. Finally, we reprove Theorem 1 of 21 and Theorem 1.6 of 2] with a much easier proof, and we also give a counter example for Question 3 of [21]. This example also says that Theorem 1.8 of [2] does not hold in general. For any unexplained notation or terminology, we refer the reader to [3] and [16].

\section{Ratliff-Rush Closure, Associated GRAded Ring}

Proposition 2.1. Let $d=2, x_{1}, x_{2}$ be a superficial sequence of $I$ and $J=\left(x_{1}, x_{2}\right)$. Let $k \in \mathbb{N}_{0}$ such that $J \cap I^{n}=J I^{n-1}$ for all $n \geq k+1$. Then $\widetilde{I^{n}}=I^{n}$ for all $n \geq 1$ if and only if $I^{n}: x_{1}=I^{n-1}$ for $n=1, \ldots, k$.

Proof. $(\Longrightarrow)$ immediately follows by [22, Corollary 2.7].

$(\Longleftarrow)$. By [22, Corollary 2.7], it is enough for us to prove $I^{n}: x_{1}=I^{n-1}$ for all $n \geq k$. By using induction on $n$, it is enough to prove the result for $n=k+1$. For this, firstly we prove that $J I^{k}: x_{1}=I^{k}$. But this is an elementary fact that $J I^{k}: x_{1}=\left(x_{1} I^{k}+x_{2} I^{k}\right): x_{1}=I^{k}+\left(x_{2} I^{k}: x_{1}\right)$ and also $x_{2} I^{k}: x_{1}=x_{2} I^{k-1}$. Hence $J I^{k}: x_{1}=I^{k}$. Therefore, by our assumption, we have $\left(J \cap I^{k+1}\right): x_{1}=I^{k}$ and so we have $I^{k+1}: x_{1}=I^{k}$, as desired.

The following result immediately follows by Proposition 2.1.

Corollary 2.2. Let $d=2, x_{1}, x_{2}$ be a superficial sequence of $I$ and $J=\left(x_{1}, x_{2}\right)$. Let $k \in \mathbb{N}_{0}$ such that $r_{J}(I)=k$. Then $\widetilde{I^{n}}=I^{n}$ for all $n \geq 1$ if and only if $I^{n}: x_{1}=I^{n-1}$ for $n=1, \ldots, k$.

Corollary 2.3. Let $d=2, x_{1}, x_{2}$ be a superficial sequence of $I$ and $J=\left(x_{1}, x_{2}\right)$ such that $r_{J}(I)=2$. Then $\widetilde{I^{n}}=I^{n}$ for all $n \geq 1$ if and only if $I^{2}: x_{1}=I$. 
The Hilbert-Samuel function of $I$ is the numerical function that measures the growth of the length of $R / I^{n}$ for all $n \in \mathbb{N}$. For all $n$ large this function $\lambda\left(R / I^{n}\right)$ is a polynomial in $n$ of degree $d$

$$
\lambda\left(R / I^{n}\right)=\sum_{i=0}^{d}(-1)^{i} e_{i}(I)\left(\begin{array}{c}
n+d-i-1 \\
d-i
\end{array}\right),
$$

where $e_{0}(I), e_{1}(I), \ldots, e_{d}(I)$ are called the Hilbert coefficients of $I$. Let $A=\bigoplus_{m \geq 0} A_{m}$ be a Notherian graded ring where $A_{0}$ is an Artinian local ring, $A$ is generated by $A_{1}$ over $A_{0}$ and $A_{+}=\bigoplus_{m>0} A_{m}$. Let $H_{A_{+}}^{i}(A)$ denote the i-th local cohomology module of $A$ with respect to the graded ideal $A_{+}$and set $a_{i}(A)=\max \left\{m \mid\left[H_{A_{+}}^{i}(A)\right]_{m} \neq 0\right\}$ with the convention $a_{i}(A)=-\infty$, if $H_{A_{+}}^{i}(A)=0$. The Castelnuovo-Mumford regularity is defined by $\operatorname{reg}(A):=\max \left\{a_{i}(A)+i \mid i \geq 0\right\}$

Proposition 2.4. Let $d=2$ and $J$ be a minimal reduction of $I$ such that $r_{J}(I)=2$. If $\widetilde{I}=I$, then we have the following:

(i) $\operatorname{reg} G(I)=2$.

(ii) $e_{2}(I)=\lambda\left(I^{2} / J I\right)$.

Proof. The case $(i)$ follows by Corollary 2.3 and [19, Theorem 2.1 and Corollay 2.2] and the case $(i i)$ follows by Corollary 2.3 and [5. Theorem 3.1].

Remark 2.5. Let $d=2, \widetilde{I}=I$ and $J$ be a minimal reduction of $I$. If $\operatorname{reg} G(I)=3$, then by [19, Lemma 1.2 and Corollary 2.2], [28, Proposition 3.2] and Proposition 2.4 we have $r_{J}(I)=3$.

The following result is an improvement of [15, Theorem 2.11] and [17, Proposition $16]$.

Proposition 2.6. Let $d=2, \widetilde{I}=I$ and $J$ be a minimal reduction of $I$. Then $r_{J}(I)=2$ if and only if $P_{I}(n)=H_{I}(n)$ for $n=1,2$, where $H_{I}(n)$ and $P_{I}(n)$ are the Hilbert-Samuel function and the Hilbert-Samuel polynomial respectively.

Proof. $(\Longrightarrow)$ let $r_{J}(I)=2$. Then by Corollary $2.3, \widetilde{I^{n}}=I^{n}$ for all $n \geq 1$ and so by [17, Proposition 16] we have $H_{I}(n)=P_{I}(n)$ for all $n=1,2$.

$(\Longleftarrow)$ is clear by [17, Proposition 16].

Remark 2.7. Let $J$ be a minimal reduction of $I, x_{1} \in J$ and $\bar{I}=I /\left(x_{1}\right), \bar{J}=J /\left(x_{1}\right)$. Then, by definition of reduction number, we have 
(i) If $r_{\bar{J}}(\bar{I})=k$ and $I^{k+1}: x_{1}=I^{k}$, then $r_{J}(I)=k$.

(ii) If $d=2$ and $I^{2}: x_{1}=I$, Then $r_{\bar{J}}(\bar{I}) \leq 2$ if and only if $r_{J}(I) \leq 2$.

Lemma 2.8. Let $d=2$ and $J$ be a minimal reduction of $I$ such that $J \cap I^{n}=J I^{n-1}$ for $n=1, \ldots, t$. If $r_{\bar{J}}(\bar{I})=k$ and $\lambda\left(I^{n+1} / J I^{n}\right)=\lambda\left(\bar{I}^{n+1} / \overline{J I}^{n}\right)$ for $n=t, \ldots, k-1$. Then $I^{n+1}: x_{1}=I^{n}$ for $n=0, \ldots, k-1$.

Proof. By [7, Proposition 1.7(ii)], $\left(x_{1}\right) \cap I^{n}=x_{1} I^{n-1}$ for $n=1, \ldots, t$ and so $I^{n}: x_{1}=$ $I^{n-1}$ for $n=1, \ldots, t$. Now, consider the exact sequence

$$
0 \longrightarrow I^{n+1}: x_{1} / J I^{n}: x_{1} \longrightarrow I^{n+1} / J I^{n} \longrightarrow \bar{I}^{n+1} / \overline{J I}^{n} \longrightarrow 0 .
$$

By our assumption, $I^{n+1}: x_{1}=J I^{n}: x_{1}$ for $n=t, \ldots, k-1$. Assume that $y x_{1} \in J I^{t}$. Then we have $y x_{1}=\alpha_{1} x_{1}+\alpha_{2} x_{2}$ for some $\alpha_{1}, \alpha_{2} \in I^{t}$. Hence $\left(y-\alpha_{1}\right) x_{1}=\alpha_{2} x_{2} \in$ $x_{2} I^{t}$ and since $x_{1}, x_{2}$ is a regular sequence, we obtain $y-\alpha_{1}=s x_{2}$ for some $s \in R$. Since $\left(y-\alpha_{1}\right) x_{1}=s x_{1} x_{2} \in x_{2} I^{t}$ and $x_{2}$ is a non-zerodivisor, it follows that $s x_{1} \in I^{t}$ and so $s \in I^{t}: x_{1}$. Therefore $s \in I^{t-1}$ and so $y \in I^{t}$. Thus by repeating this argument, we obtain $I^{n+1}: x_{1}=I^{n}$ for $n=0, \ldots, k-1$, as desired.

The following result was proved in [14, Theorem 2.4], 4, Theorem 3.10] and [25, Theorem 3.7], and we give a simplified proof.

Proposition 2.9. Let $J$ be a minimal reduction of $I$ such that $J \cap I^{n}=J I^{n-1}$ for $n=1, \ldots, t$ and $\lambda\left(I^{t+1} / J I^{t}\right) \leq 1$. Then $\operatorname{depth} G(I) \geq d-1$.

Proof. By using Sally's descent, we may deduce the problem to the case of $d=2$. Set $r_{\bar{J}}(\bar{I})=k$. Then, by using the exact sequence $(\dagger)$, we have $\lambda\left(\bar{I}^{n+1} / \overline{J I}^{n}\right)=$ $\lambda\left(I^{n+1} / J I^{n}\right) \leq 1$ for $n=t, \ldots, k-1$. By Lemma 2.8, we have $I^{n+1}: x_{1}=I^{n}$ for $n=0, \ldots, k-1$. By [14, Proposition 1.1], we know that $\sum_{n \geq 0} \lambda\left(\widetilde{I^{n+1}} / J \widetilde{I^{n}}\right)=e_{1}(I)=$ $e_{1}(\bar{I})=\sum_{n=0}^{k-1} \lambda\left(I^{n+1} / J I^{n}\right)=\sum_{n=0}^{t-1} \lambda\left(I^{n+1} / J I^{n}\right)+k-t$. Therefore by [24, Theorem 1.3], we have $r_{J}(I) \leq k$. Thus by Lemma 2.8 and Corollary 2.2 , we obtain $\widetilde{I^{n}}=I^{n}$ for all $n \geq 1$. Hence $\operatorname{depth} G(I) \geq 1$, as required.

Lemma 2.10. Let $d=2$ and $J=\left(x_{1}, x_{2}\right)$ a minimal reduction of $I$ such that $J \cap I^{n}=J I^{n-1}$ for all $n \geq 3$. If either $I^{2}: x_{1}=I$ or $I^{2}: x_{2}=I$, then $\widetilde{I^{n}}=I^{n}$ for all $n \geq 1$. In particular depth $G(I) \geq 1$.

Proof. By using the same argument that was used in the proof of proposition 2.1, the result immediately follows. 
Lemma 2.11. Let $d=2$ and $J=\left(x_{1}, x_{2}\right)$ be a minimal reduction of $I$ such that $\lambda\left(J \cap I^{2} / J I\right) \leq 1$. Then either $I^{2}: x_{1}=I$ or $I^{2}: x_{2}=I$.

Proof. If $\lambda\left(J \cap I^{2} / J I+I^{2} \cap\left(x_{1}\right)\right)=1$, then $I^{2} \cap\left(x_{1}\right) \subseteq J I$ and so $I^{2} \cap\left(x_{1}\right) \subseteq\left[x_{1} I+\right.$ $\left.x_{2} I\right] \cap\left(x_{1}\right)$. Therefore $I^{2} \cap\left(x_{1}\right)=x_{1} I$ and so $I^{2}: x_{1}=I$. If $\lambda\left(J \cap I^{2} / J I+I^{2} \cap\left(x_{1}\right)\right)=$ 0 , then $I^{2} \cap\left(x_{1}\right)+I x_{2}=J \cap I^{2}$. Hence $I^{2} \cap\left(x_{1} x_{2}\right)+I x_{2}=I^{2} \cap\left(x_{2}\right)$ and so $I x_{2}=I^{2} \cap\left(x_{2}\right)$. Thus $I^{2}: x_{2}=I$.

The following result was proved in [11, Theorem 3.2] and [12, Corollary 1.5] and we give an easier proof

Proposition 2.12. Let $J$ be a minimal reduction of $I$ such that $J \cap I^{n}=J I^{n-1}$ for all $n \geq 3$. If $\lambda\left(J \cap I^{2} / I J\right) \leq 1$, then $\operatorname{depth} G(I) \geq d-1$.

Proof. By Sally's descent, we may assume that $d=2$. Now, by using Lemmas 2.11 and 2.10 the result follows.

Theorem 2.13. Let $d \geq 3$ and $k \in \mathbb{N}_{0}$ such that $\widetilde{I^{k}}=I^{k}$. If $x_{1}, \ldots, x_{d}$ is a tame superficial sequence of $I$ and $J=\left(x_{1}, \ldots, x_{d}\right)$ such that $J \cap I^{n}=J I^{n-1}$ for all $n \geq k+2$, then $\mathfrak{a}^{m} I^{n}: x_{1}=\mathfrak{a}^{m} I^{n-1}$ for all $n \geq k+1$ and all $m \in \mathbb{N}_{0}$, where $\mathfrak{a}=\left(x_{2}, \ldots, x_{d}\right)$. In particular, $\widetilde{I^{n}}=I^{n}$ for all $n \geq k$.

Proof. We will proceed by induction on $n$. Assume $n=k+1$. Then by [18, Lemma 2.7] and our assumption we have $\mathfrak{a}^{m} I^{k+1}: x_{1} \subseteq \mathfrak{a}^{m} \widetilde{I^{k+1}}: x_{1}=\mathfrak{a}^{m} \widetilde{I^{k}}=\mathfrak{a}^{m} I^{k}$. Therefore $\mathfrak{a}^{m} I^{k+1}: x_{1}=\mathfrak{a}^{m} I^{k}$ for all $m \in \mathbb{N}_{0}$. Assume $n \geq k+1$ and that for all $t$ with $k+1 \leq t \leq n$ and all $m \in \mathbb{N}_{0}, \mathfrak{a}^{m} I^{t}: x_{1}=\mathfrak{a}^{m} I^{t-1}$. We show that for all $m \in \mathbb{N}_{0}, \mathfrak{a}^{m} I^{n+1}: x_{1}=\mathfrak{a}^{m} I^{n}$. Let $y x_{1}$ be an element of $\mathfrak{a}^{m} I^{n+1}$. Then $y x_{1} \in \mathfrak{a}^{m}$ and by using [18, Lemma 2.1] we obtain $y \in \mathfrak{a}^{m}$. Therefore we can write the expression, $y=\sum_{i_{2}+\ldots+i_{d}=m} r_{i_{2} \ldots i_{d}} x_{2}^{i_{2}} \ldots x_{d}^{i_{d}}$. Since the element $y x_{1}$ belongs to $\mathfrak{a}^{m} I^{n+1}$ too, we obtain the following equalities

$$
\sum_{i_{2}+\ldots+i_{d}=m} r_{i_{2} \ldots i_{d}} x_{1} x_{2}^{i_{2}} \ldots x_{d}^{i_{d}}=y x_{1}=\sum_{i_{2}+\ldots+i_{d}=m} s_{i_{2} \ldots i_{d}} x_{2}^{i_{2}} \ldots x_{d}^{i_{d}},
$$

where $s_{i_{2} \ldots i_{d}} \in I^{n+1}$ for all $i_{2}, \ldots, i_{d}$ such that $i_{2}+\ldots+i_{d}=m$. As $x_{1}, \ldots, x_{d}$ is a regular sequence in $R$, by equating coefficients in the previous expressions, we get $r_{i_{2} \ldots i_{d}} x_{1}-s_{i_{2} \ldots i_{d}} \in\left(x_{2}, \ldots, x_{d}\right)$ for all $i_{2}, \ldots, i_{d}$ such that $i_{2}+\ldots+i_{d}=m$. Hence $s_{i_{2} \ldots i_{d}} \in J \cap I^{n+1}$ and by our assumption we obtain $s_{i_{2} \ldots i_{d}} \in J I^{n}$ for all $i_{2}, \ldots, i_{d}$ such 
that $i_{2}+\ldots+i_{d}=m$. Hence, going back to the equalities we wrote for $y x_{1}$, we obtain $y x_{1} \in \mathfrak{a}^{m} J I^{n}=\mathfrak{a}^{m+1} I^{n}+x_{1} \mathfrak{a}^{m} I^{n}$. Therefore we have

$$
\mathfrak{a}^{m} I^{n+1} \cap\left(x_{1}\right) \subseteq \mathfrak{a}^{m+1} I^{n} \cap\left(x_{1}\right)+x_{1} \mathfrak{a}^{m} I^{n}=x_{1}\left(\mathfrak{a}^{m+1} I^{n}: x_{1}\right)+x_{1} \mathfrak{a}^{m} I^{n} .
$$

By applying the inductive hypothesis we get $\mathfrak{a}^{m} I^{n+1} \cap\left(x_{1}\right) \subseteq x_{1} \mathfrak{a}^{m+1} I^{n-1}+x_{1} \mathfrak{a}^{m} I^{n}=$ $x_{1} \mathfrak{a}^{m} I^{n}$. This proves that $\mathfrak{a}^{m} I^{n+1}: x_{1} \subseteq \mathfrak{a}^{m} I^{n}$ and so $\mathfrak{a}^{m} I^{n+1}: x_{1}=\mathfrak{a}^{m} I^{n}$ for all $m \in \mathbb{N}_{0}$. In particular, if we set $m=0$, then $I^{n+1}: x_{1}=I^{n}$ for all $n>k$ and so by [22, Corollary 2.7], $\widetilde{I^{n}}=I^{n}$ for all $n \geq k$, as desired.

The following result easily follows by Theorem 2.13 .

Corollary 2.14. Let $x_{1}, \ldots, x_{d}$ be a tame superficial sequence of $I$ and $J=$ $\left(x_{1}, \ldots, x_{d}\right)$.

(i) If $\widetilde{I}=I$ and $J \cap I^{n}=J I^{n-1}$ for all $n \geq 3$, then $\widetilde{I^{n}}=I^{n}$ for all $n \geq 1$. In particular depth $G(I) \geq 1$.

(ii) If $r_{J}(I)=2$, then $\widetilde{I}=I$ if and only if $\operatorname{depth} G(I) \geq 1$.

(iii) Let $k \in \mathbb{N}_{0}$ such that $r_{J}(I)=k+1$ and $\widetilde{I^{k}}=I^{k}$. Then $\widetilde{I^{n}}=I^{n}$ for all $n \geq k$.

The following example shows that the equality of Corollary 2.14(ii) maybe happen.

Example 2.15. Let $K$ be a field, $R=K \llbracket x, y \rrbracket, I=\left(x^{6}, x^{4} y^{2}, x^{3} y^{3}, x^{2} y^{4}, x y^{5}, y^{6}\right)$ and $J=\left(x^{6}, y^{6}+x^{4} y^{2}\right)$. Then $r_{J}(I)=2$, depth $G(I)=1$ and so $G(I)$ is not C.M.

\section{INVARIANCE OF A LENGTH}

Let $J=\left(x_{1}, \ldots, x_{d}\right)$ be a minimal reduction of $I$. In [29] Wang defined the following exact sequence for all $n, k$

$$
0 \longrightarrow T_{n, k} \longrightarrow \oplus^{\left(\begin{array}{c}
k+d-1 \\
d-1
\end{array}\right)} I^{n} / J I^{n-1} \stackrel{\phi_{k}}{\longrightarrow} J^{k} I^{n} / J^{k+1} I^{n-1} \longrightarrow 0
$$

where $\phi_{k}=\left(x_{1}^{k}, x_{1}^{k-1} x_{2}, \ldots, x_{1}^{k-1} x_{d}, \ldots, x_{d}^{k}\right)$ and $T_{n, k}=\operatorname{ker}\left(\phi_{k}\right)$. He also showed that $T_{1, k}=0$ for all $k$ and if $d=1$, then $T_{n, k}=0$ for all $n, k$. By using the exact sequence $(*)$, we drive the following easy lemma and we leave the proof to the reader.

Lemma 3.1. Let $t \in \mathbb{N}_{0}$ and $J=\left(x_{1}, \ldots, x_{d}\right)$ be a minimal reduction of $I$. Then we have the following:

(i) If $J \cap I^{n}=J I^{n-1}$ for $n=1, \ldots, t$, then $T_{n, k}=0$ for $n=1, \ldots, t$ and all $k$. 
(ii) If I is integrally closed, then $T_{2, k}=0$ for all $k$. In particular, if $I=m$, then $T_{2, k}=0$ for all $k$.

The following lemma is known see the proof of [4, Proposition 2.1].

Lemma 3.2. Let $J=\left(x_{1}, \ldots, x_{d}\right)$ be a minimal reduction of $I$. Then

$\lambda(I / J)=e_{0}(I)-\lambda(R / I)$ and $\lambda\left(I^{n+1} / J^{n} I\right)=e_{0}(I)\left(\begin{array}{c}n+d-1 \\ d\end{array}\right)+\lambda(R / I)\left(\begin{array}{c}n+d-1 \\ d-1\end{array}\right)-$ $\lambda\left(R / I^{n+1}\right)$ for $n \geq 1$ which are independent of $J$.

In [21, Puthenpurakal proved that $\lambda\left(\mathfrak{m}^{3} / J^{2}{ }^{2}\right)$ is independent of the minimal reduction $J$ of $\mathfrak{m}$ and subsequently Ananthnarayan and Huneke [2] extend it for $n$-standard admissible $I$-filtrations.

The following result was proved in [21, Theorem 1] and [2, Theorem 3.5]. We reprove it with a much easier proof.

Theorem 3.3. Let $t \in \mathbb{N}_{0}$ and $J=\left(x_{1}, \ldots, x_{d}\right)$ be a minimal reduction of $I$. If $J \cap I^{n}=J I^{n-1}$ for $n=1, \ldots, t$, then $\lambda\left(I^{n+1} / J I^{n}\right)$ is independent of $J$ for $n=1, \ldots, t$.

Proof. We have $\lambda\left(I^{n+1} / J I^{n}\right)=\lambda\left(I^{n+1} / J^{n} I\right)-\sum_{k=1}^{n-1} \lambda\left(J^{k} I^{n+1-k} / J^{k+1} I^{n-k}\right)$. Therefore by Lemma 3.1 and the exact sequence $(*)$, we obtain $\lambda\left(I^{n+1} / J I^{n}\right)=\lambda\left(I^{n+1} / J^{n} I\right)-$ $\sum_{k=1}^{n-1}\left(\begin{array}{c}k+d-1 \\ d-1\end{array}\right) \lambda\left(I^{n+1-k} / J I^{n-k}\right)$. Now by using Lemma 3.2 and using induction on $n$, the result follows.

The following example is a counterexample for Question 3 of [21] and it also says that Theorem 1.8 of 2] does not hold in general. The computations are performed by using Macaulay2 [9], CoCoA [1] and Singular [10].

Example 3.4. Let $K$ be a field and $S=K \llbracket x, y, z, u, v \rrbracket$, where $I=\left(x^{2}+y^{5}, x y+\right.$ $\left.u^{4}, x z+v^{3}\right)$. Then $R=S / I$ is a Cohen-Macaulay local ring of dimension two, ideals $J_{1}=(y, z) R$ and $J_{2}=(z, u) R$ are minimal reduction of $\mathfrak{m}=(x, y, z, u, v) R$ and $\lambda\left(\mathfrak{m}^{4} / J_{1} \mathfrak{m}^{3}\right)=17, \lambda\left(\mathfrak{m}^{4} / J_{2} \mathfrak{m}^{3}\right)=20$.

Acknowledgement . This paper was done while I was visiting the University of Osnabruck. I would like to thank the Institute of Mathematics of the University of Osnabruck for hospitality and partially financial support and I also would like to express my very great appreciation to Professor Winfried Bruns for his valuable and constructive suggestions during the planning and development of this research work. Moreover, I would like to thank deeply grateful to the referee for the careful reading of the manuscript and the helpful suggestions. 


\section{REFERENCES}

[1] J. Abbott and A. M. Bigatti, a C++ library for doing Computations in Commutative Algebra, Available at http://cocoa.dima.unige.it/cocoalib.

[2] H. Ananthnarayan and C. Huneke, 3-standardness of the maximal ideal, J. Pure and Appl. Algebra, 215(2011), 2674-2683.

[3] W. Bruns and J. Herzog, Cohen-Macaulay rings, Cambridge University Press, Cambridge, UK, 1998.

[4] A. Corso, C. Polini and M. Vaz Pinto, Sally modules and associated grade rings, Comm. Algebra, 26(1998), 2689-2708.

[5] A. Corso, C. Polini and M. E. Rossi, Depth of associated graded rings via Hilbert coefficients of ideals, J. Pure and App. Algebra, 201(2005), 126-141.

[6] T. T. Dinh, M. E. Rossi and N. V. Trung, Castelnuovo-Mumford regularity and Ratliff-Rush closure, arXiv: 1512.04372 .

[7] J. Elias, Depth of higher associated graded rings, J. London Math. Soc., 70(2004), 41-58.

[8] J. Elias, On the computation of the Ratliff-Rush closure, J. Symbolic Comput., 37(2004), 717-725.

[9] D. R. Grayson and M. E. Stillman, Macaulay 2, a software system for research in algebraic geometry, Available at http://www.math.uiuc.edu/Macaulay2.

[10] G. M. Greuel, S. Laplagne and G. Pfister, normal.lib. A Singular 4-0-2 library for computing the normalization of affine rings (2015).

[11] A. Guerrieri, On the depth of the associated graded ring of an $\mathfrak{m}$-primary ideal of a CohenMacaulay local ring, J. Algebra, 167(1994), 745-757.

[12] A. Guerrieri and M. E. Rossi, Estimates on the depth of the associated graded ring, J. Algebra, 211(1999), 457-471.

[13] W. Heinzer, D. Lantz and K. Shah, The Ratliff-Rush ideals in a Noetherian ring, Comm. Algebra, 20(1992), 591-622.

[14] S. Huckaba, On the associated graded rings having almost maximal depth, Comm. Algebra, 26(1998), 967-976.

[15] C. Huneke, Hilbert functions and symbolic powers. Michigan. Math. J., 34(1987), 293-318.

[16] C. Huneke and I. Swanson, Integral closure of ideals, rings and modules. Cambridge University Press, Cambridge, UK, 2006.

[17] S. Itoh, Hilbert coefficients of integrally closed ideals, J. Algebra, 176(1995), 638-652.

[18] A. Mafi, Ratliff-Rush ideal and reduction numbers, arXiv: 1510.02278v1.

[19] T. Marley, The reduction number of an ideal and the local cohomology of the associated graded ring, Proc. Amer. Math. Soc., 117(1993), 335-341.

[20] D. G. Northcott and D. Rees, Reduction of ideals in local rings, Math. Proc. Cambridge Philos. Soc., 50(1954), 145-158.

[21] T. J. Puthenpurakal, Invariance of a length associated to a reduction, Comm. Algebra, 33(2005), 2039-2042. 
[22] T. J. Puthenpurakal, Ratliff-Rush filtration, regularity and depth of higher associated graded modules I, J. Pure Appl. Algebra, 208(2007), 159-176.

[23] L. J. Ratliff and D. Rush, two notes on reductions of ideals, Indiana Univ. Math. J., 27(1978), 929-934.

[24] M. E. Rossi, A bound on the reduction number of a primary ideal, Proc. Amer. Math. Soc., 128(2000), 1325-1332.

[25] M. E. Rossi, Primary ideals with good associated graded ring, J. Pure and Appl. Algebra, 145(2000), 75-90.

[26] J. D. Sally, Hilbert coefficients and reduction number 2, J. Alg. Geometry, 1(1992), 325-333.

[27] I. Swanson, A note on analytic spread, Comm. Algebra, 22(1994), 407-411.

[28] N. V. Trung, Reduction exponent and dgree bound for the defining equations of graded rings, Proc. Amer. Math. Soc., 101(1987), 229-236.

[29] H. Wang, An interpretation of $\operatorname{depth}(G(I))$ and $e_{1}(I)$ via the Sally module, Comm. Algebra, 25(1997), 303-309.

A. Mafi, Department of Mathematics, University of Kurdistan, P.O. Box: 416, SANANDAJ, IRAN.

E-mail address: a_mafi@ipm.ir 